\title{
Acute Myocradial Infarction, Primary Percutanious Coronary Intervention (PCI) in an Anticoagulated Patient for Mitalic Mitral Valve Replacement (MVR) Aeham Rabadi*1, Mohammad Alsmadi², Rayya Almashagbeh ${ }^{1}$, Yazan Banihamad ${ }^{1}$, Tareq AL-Qudah ${ }^{1}$ \\ 1-Cardiology department, Queen Alia Heart Institute (QAHI), Royal Medical Services (RMS), Jordan \\ 2-Gastroentrology Department, King Husain Medical City, Royal Medical Services (RMS), Jordan \\ *Corresponding Author: Dr. Aeham Rabadi, Email: ayhamrabai84@gmail.com,
} Mobile: 00962772292924

\begin{abstract}
Valvular heart diseases and atrial fibrillation are ones of the most common indications for oral anticoagulation, in the same time they are known risk factors for coronary artery disease and presentation of acute coronary syndrome, which may need urgent management by primary percutaneous intervention.

Acute myocardial infarction is commonly seen in these patients with suboptimal anticoagulation, but it is almost seldomly seen when anticoagulation is in therapeutic range.

This is the first case reporting an acute myocardial infarction (MI) in a patient post mitral valve replacement (MVR) and high International Normalization Ratio (INR), which has not been reported before.
\end{abstract}

Keywords: Acute myocardial infarction; Major bleeding; Percutaneouscoronary intervention; Warfarin.

\section{INTRODUCTION}

Acute myocardial infarction (MI) is a diagnosis of acute myocardial injury thatoccursdue to acute mismatch between cardiac oxygen demand and blood supply caused mostly secondary to coronary occlusion. It is clinically defined as the presence of prolonged severe chest pain or any other equivocal symptom or sign such as ST-elevation on electrocardiography (ECG) with rise in specific cardiac enzymes and the development of $\mathrm{Q}$ wave in at least two consecutive leads ${ }^{(1)}$.

The presentation of acute MI post valvular replacement is very rare and mostly is secondary to suboptimal anticoagulation. The first case of acute inferior MI in a patient with a prosthetic aortic valve and high international normalized ratio was published on 23 March2014, which was not reported previously, and there was no other report for any similar case after that ${ }^{(2)}$.

This is the first case reporting an acute MI in a patient post MVR and high International Normalization Ratio (INR).

\section{CASE REPORT}

A 56 years old male patient ex-smoker for 15 years and a known case of mitral stenosis who underwent MVR and tricuspid valve (TV) repair in
2014 after a selective coronary angiography, which showed nonocclusive mild coronary artery disease (CAD), presented to the emergency room with retrosternal severe chest pain.

He was stable with blood pressure of 120/80 and a heart rate of 60 beets /minute. ECG showed acute inferoposterior S-T segment elevation myocardial infarction (STEMI)and echocardiography showed a normal left ventricular function with wellfunctioning MVR without any noticed complications over the metallic valve .

The patient was on aspirin $81 \mathrm{mg}$ and warfarin $7.5 \mathrm{mg}$ daily, atorvastatin $20 \mathrm{mg}$, metoprolol $50 \mathrm{mg}$ and enalapril $2.5 \mathrm{mg}$ twice daily.

Laboratory results showed normal kidney and liver function tests and normal CBC with creatine phosphokinase (CPK) $350 \mathrm{U} / \mathrm{L}$ and INR 3.9. Patient was taken to catheter lab after loading him with clopidogrel $600 \mathrm{mg}$ orally, no other anticoagulant was given before catheterization.

Catheterization was done via the transfemoral access according to patientpreference, and periprocedural heparin $5000 \mathrm{IU}$-IV was given before stenting the right coronary artery (RCA) as seen in figure 1 , which shows the long occlusion of mid RCA and how it was opened by primary PCI. 


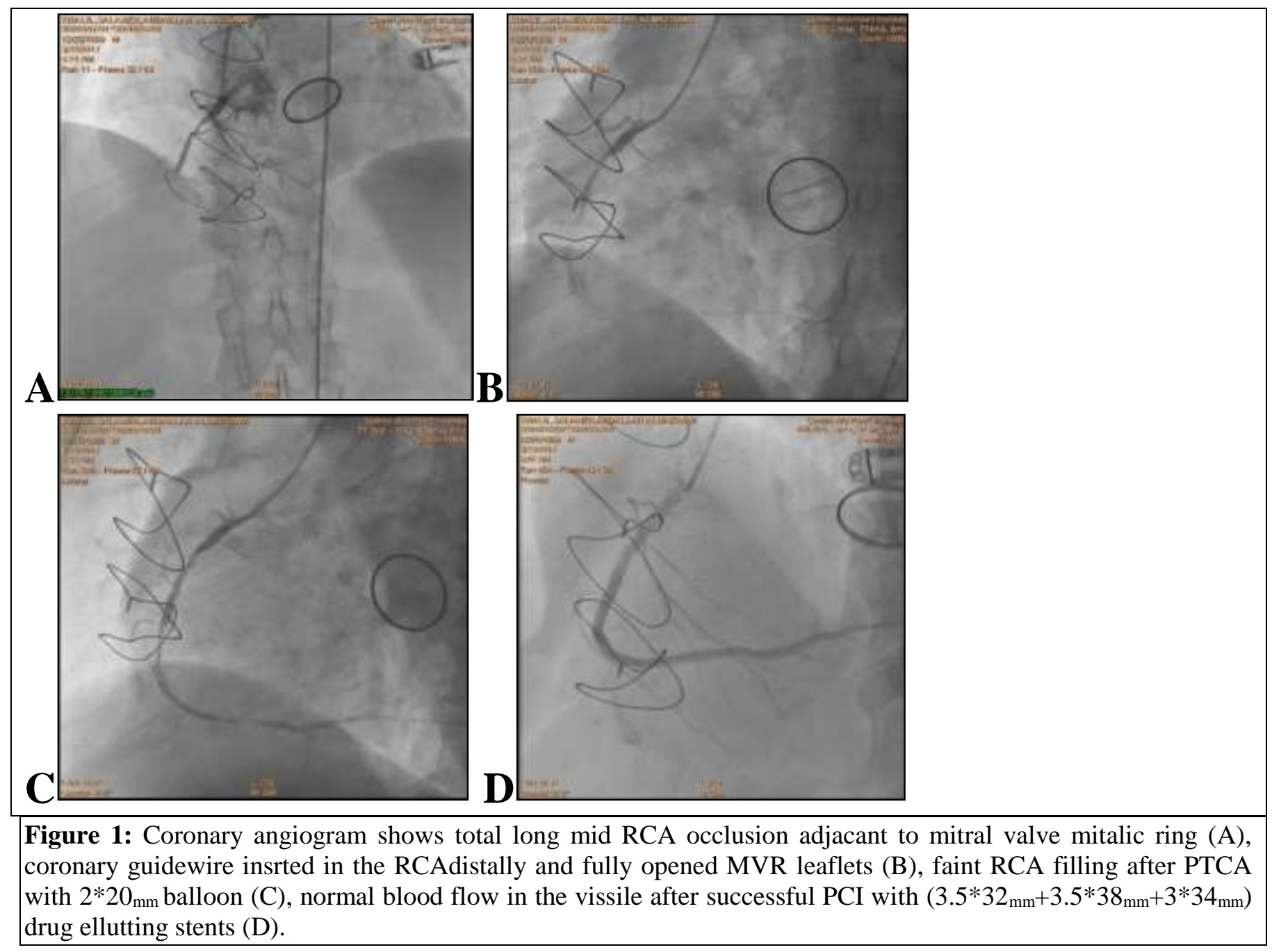

Site of puncture was closed by angioseal and warfarin was held till INR less than 3. No major bleeding or vascular complications happened during his hospital stay, and after 5 days the patient was discharged on triple therapy (dual antiplatelets and warfarin) for one month then aspirin $81 \mathrm{mg}$ was stopped after a month.

After one year clopidogrel was stopped and aspirin $81 \mathrm{mg}$ was reinitiated after an elective catheterization which showed patent RCA stents.

\section{DISCUSSION}

Most of the studies and researches discussed the risk of Acute Coronary Syndrome (ACS) and MI in patients with atrial fibrillation (AF) who are anticoagulated. They compared vitamin $\mathrm{K}$ antagonist (warfarin) with different direct oral anticoagulants (NOACs). According to which the latter were more preventive of acute coronary events. On the other hand no study has addressed the risk of acute coronary events post valvular replacement with optimal anticoagulation and how to manage them; that is because $\mathrm{AF}$ is a known risk factor of acute $\mathrm{MI}$ and increases morbidity and mortality in these patients even if they are properly anticoagulated ${ }^{(1,3)}$.
Patients on warfarin with prior history of CAD are more prone for acute cardiovascular events and sudden death than those who had no previous coronary event ${ }^{(1)}$.

One of the major studies was published in 2012. It was one of the primary studies depending on the National Cardiovascular Data Registry, that discussed risk of bleeding in patients presented with ACS - Non S-T segment Elevation MI (NSTEMI), this study showed that risk of bleeding in these patients are higher after starting antithrombotic and antiplatelets therapy irrespective of the INR readings ${ }^{(4)}$.

On 8 April 2019 the American Heart Association Journal (JAHA) published an article discussing the outcome of In-hospital bleeding in MI patients that are previously on warfarin or direct oral anticoagulants, this study showed no major difference in the risk of major bleeding between those who are on anticoagulation before MI and those who are not, but they found that door to balloon time was longer in STEMI patients who are on home anticoagulation ${ }^{(5)}$.

American College of Cardiology published the latest review article on Oct 22, 2015 about MI and primary PCI management in patients on oral anticoagulation to decrease risk of bleeding and 
preserve optimal anticoagulation for risk of thrombosis, the recommendations were: 1)The radial arterial access is preferred over the femoral access as the former reduced bleeding and vascular complications ,2) In warfarin era no need for periprocedural anticoagulation if INR is in the therapeutic range or above but still heparin and bivalirudin can be given especially if guided by the activated clotting time (ACT) but not to give glycoprotein IIb/IIIa inhibitors, 3)A loading dose of clopidogrel $600 \mathrm{mg}$ with aspirin $325 \mathrm{mg}$ is preferred over the newer antiplatelet agents to lower the risk of bleeding, and finally 4)To shorten the period of triple therapy (dual antiplatelets and warfarin) to one month then to keep on clopidogrel and warfarin for another 11 months till safe discontinuing of clopidogrel and resuming aspirin $81 \mathrm{mg}^{(6)}$.

\section{CONCLUSION}

Patients on oral anticoagulantswith STEMI undergoing primary PCI pose a challenging clinical situation to interventional cardiologists. The current literature is limited in the management of these patients in terms of choosing the appropriate antiplatelet and anticoagulation regimens to balance the risk of ischemic events and bleeding.

Until further data are available, use of radial access, loading dose of aspirin and clopidogrel, and additional peri-procedural anticoagulation with heparin or bivalirudin in the acute setting for these patients is recommended.

Post-ACS: for patients at high risk of embolic events, triple therapy (dual antiplatelets plus warfarin or reduced-dose of NOACs) is recommended for leastduration possible.

\section{REFERENCES}

1. Stefan H.H, Jonas O, Sean Y et al. (2012): Myocardial ischemic events in patients with atrial fibrillation treated with dabigatran or warfarin in the RE-LY (Randomized Evaluation of Long-Term Anticoagulation Therapy) Trial. Circulation, 125:669676.

2. Halil A, Ibrahim S, Kenan D et al. (2014): Acute inferior myocardial infarction in a patient with a prosthetic aortic valve and high international normalized ratio. Postep Kardiol Inter., 10 (35): 63-65.

3. Christina Ji-Young L, Thomas A G, Nicholas $\mathrm{C}$ et al. (2018):Risk of myocardial infarction in anticoagulated patients with atrial fibrillation. J Am Coll Cardiol., 72 (1): 17-26.

4. Sumeet S, Eric D et al. (2012): admission international normalized ratio levels, early treatment strategies, and major bleeding risk among non-STSegment-elevation myocardial infarction patients on home warfarin therapy: Insights from the NationalCardiovascular Data Registry. Circulation., 125:1414-1423.

5. Dmitriy $N$, Tracy $Y$ et al. (2019):In- Hospital Bleeding outcomes of myocardial infarction in the era of warfarin and direct oral anticoagulants for atrial fibrillation in the United States: A report from the National Cardiovascular Data Registry Acute Coronary Treatment and Intervention Outcomes Network Registry. Journal of the American Heart Association., 8:e011606.

6. Jaya R , Amir S (2015): Management of STEMI in patients on NOACs and undergoing primary PCI. ACC, Latest in Cardiology, Expert Analysis.https://www.acc.org/latest-incardiology/articles/2015/10/22/10/43/management-ofstemi-in-patients-on-noacs-and-undergoing-primarypci 